\title{
De la connaissance des espèces à la gestion des espaces : l'action territorialisée d'un parc national
}

\author{
Thomas Debril \\ Sociologue, INRA, UMR AGIR, 31320 Castanet Tolosan, France
}

L'action territorialisée de l'action publique représente un tournant que la sociologie des organisations comme des politiques publiques a identifié depuis longtemps. Tout en s'inscrivant dans cette perspective, cet article propose une lecture fine des conséquences de l'action territorialisée qui démontre que, loin de représenter un processus univoque, elle est l'objet de réinterprétations et d'infléchissements qui restituent la complexité des questions territoriales. L'action territorialisée est par excellence une action située dont on ne peut comprendre les logiques pouvant donner lieu à des configurations très contrastées que si on restitue le lien entre contexte et régime d'action. De ce point de vue, cet article pose la question de l'exercice de la souveraineté de l'État à partir du moment où il n'est plus le seul porteur de sens et, a fortiori, où il semble faire écho à la pluralité des situations.

La Rédaction

\section{Mots-clés :}

politique publique ; environnement ; territoire ; négociation ; parc national

\section{Keywords:}

policy; environment; territory; negotiation; national park

Résumé - L'article porte sur la mise en œuvre des politiques environnementales à partir du cas du parc national de la Vanoise. Sur la base d'un travail de comparaison entre deux vallées, la Maurienne et la Tarentaise, l'article montre d'abord comment les usagers de cet espace protégé, en surmontant le caractère problématique de leur coopération, font émerger deux formes d'organisation contrastées de leurs territoires. Il explique ensuite comment ces deux formes d'organisation conditionnent la portée de la politique du parc et comment les agents de terrain influent localement sur les pratiques qui y ont cours. La coexistence de ces deux formes d'action publique territorialisées, si elle nous montre comment sont négociés localement les enjeux environnementaux, interroge toutefois la cohérence des décisions du parc et n'est pas sans créer d'importantes tensions au sein de l'établissement.

\begin{abstract}
From the knowledge of species to the management of space: the scalar politics of a national park implementation. The article deals with the implementation of environmental policies taking as an example the case of the national park of Vanoise. Comparing the case studies of the Maurienne and Tarentaise valley, the paper shows how the users of this protected space, create two contrasted organizational frameworks of their territories by surmounting the problematic character of their cooperation. It also discloses how these two organizational frameworks condition the impact of the policy of the park and how the agents influence the local practices. The coexistence of these two forms of territorialized public action, shows us how is locally negotiated the environmental stakes. It questions however the coherence of the decisions of the park and creates important tensions within the organization.
\end{abstract}

Le parc national de la Vanoise semble à un moment crucial de son histoire. Depuis sa création, en 1963, l'entrée du parc sur le terrain s'est d'abord concentrée

sur la zone centrale ${ }^{1}$ (Fig.). Le marquage des frontières et le quadrillage de l'espace, l'activisme réglementaire et la police de l'environnement, comme la production de

\footnotetext{
Auteur correspondant : thomas.debril@toulouse.inra.fr

1 Précisons ici que le parc national de la Vanoise, situé dans le département de la Savoie, est divisé en deux grandes vallées, la Tarentaise et la Maurienne, qui comportent une richesse floristique et faunistique hors du commun. Le parc est par ailleurs divisé en deux zones, la zone centrale de $535 \mathrm{~km}^{2}$ et la zone périphérique de $1465 \mathrm{~km}^{2}$, récemment rebaptisées respectivement cœur et aire optimale d'adhésion. Ce travail s'appuie en partie sur les données empiriques récoltées lors des chantiers de recherche du master de sociologie de l'action de Sciences Po et sur le travail mené par H. Bertillo, A. Jourdain, N. Lévêque, M. Toqué. Il a par ailleurs bénéficié des commentaires de M. Mormont. Qu'ils trouvent ici l'expression de mes remerciements. Ce travail a également fait l'objet d'une présentation lors des troisièmes journées de recherches en sciences sociales Inra- Sfer-Cirad des 9, 10 et 11 décembre 2009 à Montpellier.
} 


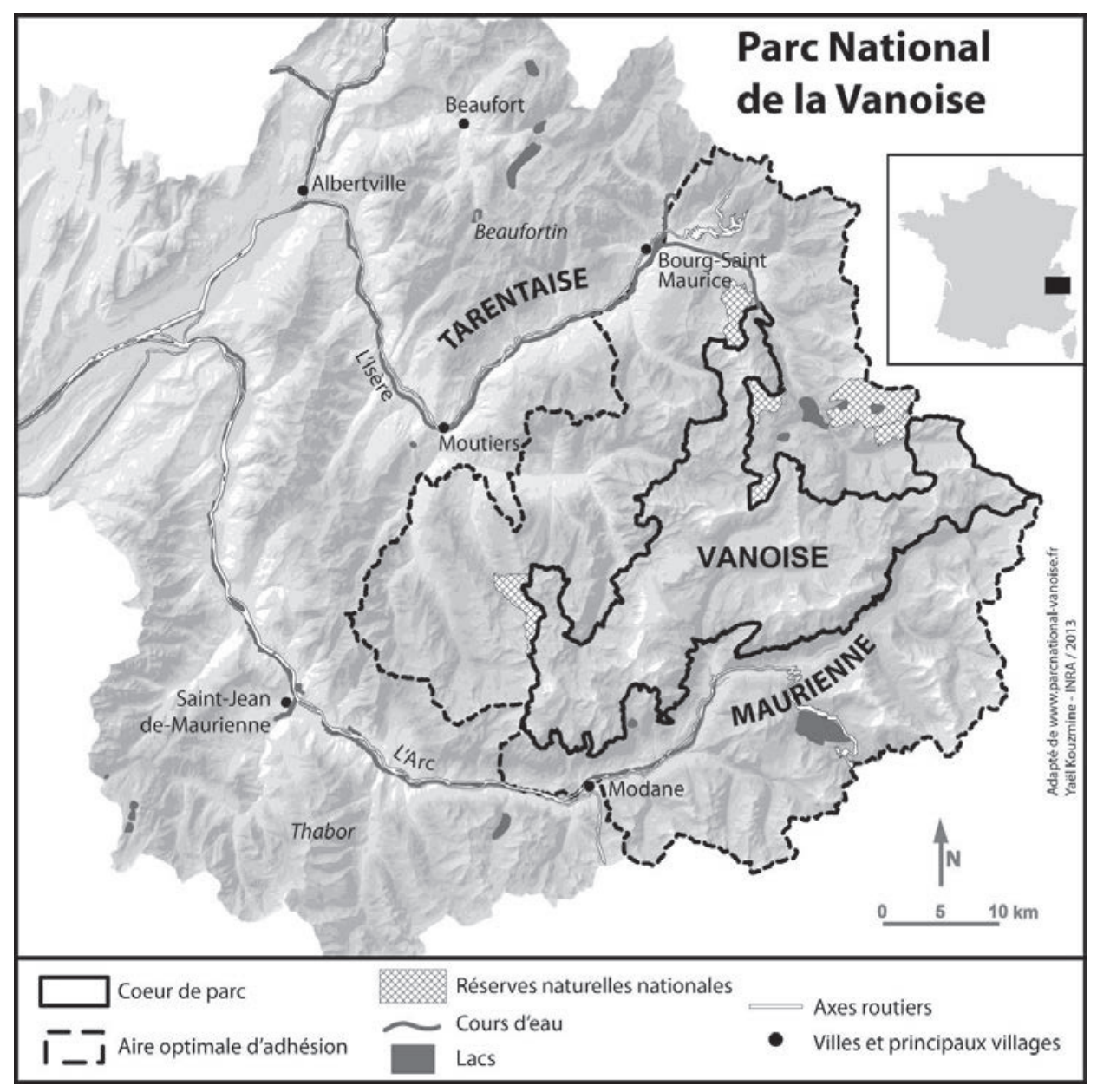

Fig. Carte du parc national de la Vanoise.

connaissances faunistiques et floristiques apparaissent alors comme les éléments marquant l'inscription du parc sur le territoire (Mauz, 2003). Pour autant, cet activisme du parc en zone centrale s'est non seulement traduit par un relatif abandon de la zone périphérique mais aussi par une relative défiance des acteurs locaux à l'égard de l'établissement. Si les parcs nationaux doivent à la fois assurer une politique de conservation et de développement rural, ce double objectif s'est d'abord traduit par un traitement géographique différencié entre zone périphérique et zone centrale (Selmi, 2006). Prenant acte de la relative défiance des acteurs locaux à l'égard de l'établissement public comme de l'ambiguïté quant au statut juridique de la zone périphérique, le député Giran a proposé de clarifier aussi bien les modes d'action des parcs que les frontières de leurs compétences (Giran, 2003). Avec la loi du 14 avril 2006, il ne s'agit plus simplement pour les chargés de mission et leurs agents de réaliser des inventaires dans la zone centrale et de sanctionner les infractions au code de l'environnement. Plus que cela, il s'agit, conformément à ce qui s'est joué dès le milieu des années 1980 dans les réserves de biosphère
(Cibien, 2006), d'enrôler les communes derrière une charte rappelant les enjeux naturalistes dans la zone périphérique et de réaffirmer la solidarité écologique entre les deux espaces (Mathevet et al., 2010).

Ce moment particulier dans l'histoire du parc nous est apparu comme un moment privilégié pour interroger, en mobilisant le mode de raisonnement proposé par la sociologie de l'action organisée (Crozier et Friedberg, 1977 ; Musselin, 2005), la mise en œuvre d'une politique de la nature qui tente de passer d'une approche scientifique et autoritaire en zone centrale à une approche gestionnaire et participative en zone périphérique. Le parc, à l'instar de son homologue cévenol (Crosnier, 2006), nous donne en effet à voir une approche partenariale soucieuse de prendre en compte, en amont, l'hétérogénéité des enjeux portés par la diversité des acteurs locaux (Delsaut et Meur-Férec, 2009), marquant ce que, à la suite des travaux de Muller (Muller, 2005), certains identifient comme un changement de référentiel dans les pratiques d'aménagement des territoires. Dans cette perspective, nous voulons montrer comment la recherche active du consentement des acteurs locaux par la direction du parc 
conduit les agents de terrain de l'établissement à développer des formes distinctes d'administration de la nature, renvoyant à des formes contrastées d'organisation de leur territoire.

Nous montrerons d'abord comment coexistent, au sein du territoire administré par le parc, deux jeux d'acteurs distincts dans les vallées de la Maurienne et de la Tarentaise. Les conflits que l'on peut observer entre ces différents acteurs, en même temps qu'ils nous révèlent comment sont négociées des règles de partage et de légitimation des usages de l'espace, nous dévoilent simultanément l'organisation spécifique de ces territoires qui conditionnent non seulement l'émergence (Boncœur et al., 2007) mais aussi la mise en œuvre des politiques qu'on souhaite leur appliquer (Duran, 1993). La comparaison de ces deux formes d'organisation territoriale nous permettra ainsi de distinguer, d'une part, un jeu social que nous qualifierons d'étanche aux injonctions du parc, la Tarentaise, dans la mesure où il n'offre que peu de prise aux agents de terrain et, d'autre part, un jeu social plus ouvert à l'administration de la nature et aux initiatives environnementales des personnels, la Maurienne.

Nous nous intéresserons alors plus directement aux pratiques des agents eux-mêmes pour montrer comment ils négocient localement leur entrée sur le terrain, composent avec l'organisation contrastée des territoires et travaillent, à l'instar des grands aménageurs analysés par Jobert (1998), à la prise en compte des enjeux environnementaux. Nous décrirons ensuite comment les agents, en travaillant l'articulation entre les injonctions de leur direction et les aspirations des acteurs locaux, donnent simultanément un contenu spécifique à l'action environnementale du parc sur le terrain. La comparaison des formes d'administration de la nature au sein des deux espaces nous permettra ainsi de distinguer, d'une part, une action à la marge des agents du parc qui coopèrent avec les acteurs périphériques de la Tarentaise et, d'autre part, une action plus engagée en Maurienne, où les agents négocient plus directement la prise en compte de leurs aspirations environnementales.

Cette action publique territorialisée (Duran et Thoenig, 1996) interroge ainsi non seulement le travail de médiation (Nay et Smith, 2002) auquel se livrent les agents articulant la diversité des enjeux locaux qu'ils rencontrent quotidiennement sur le terrain et les aspirations de leur direction plus éloignée, à Chambéry, mais questionne aussi plus directement la cohérence et la légitimité des arbitrages rendus par le siège. On comprend que cette action publique dédoublée du parc, en même temps qu'elle assure son efficacité par une adaptation aux singularités du terrain, échappe à sa direction et suscite d'importantes controverses au sein de l'établissement.

\section{La Tarentaise, étanchéité du territoire et action à la marge}

L'organisation socioéconomique de cette vallée apparaît largement structurée par l'arrangement que l'on peut observer entre les maires des communes et les exploitants des domaines skiables. Il est vrai que 449 des 520 remontées mécaniques que compte la Vanoise, sont implantées en Tarentaise et représentent 4195 ha de pistes pour une longueur estimée à $1066 \mathrm{~km}$. Cette vallée, hautement spécialisée dans les sports d'hiver, accueille ainsi un grand nombre de touristes au sein d'importantes stations comme Les Ménuires, Val Thorens, Courchevel ou La Plagne, et compte de nombreuses structures d'hébergement puisque près de $82 \%$ de la capacité d'accueil touristique se trouve en Tarentaise. Dans cette perspective, nous montrerons que l'importance de l'économie touristique associée aux domaines skiables place non seulement les agriculteurs dans une position de plus en plus difficile, mais fabrique aussi la relative étanchéité de ce territoire aux préoccupations environnementales portées par le parc.

\section{Quand les agriculteurs paient les coûts de la coopération entre exploitants de domaines skiables et élus locaux}

Aux yeux des élus, le tourisme d'hiver et les stations de ski apparaissent d'abord comme le moyen de développer efficacement l'activité économique de leurs communes. Les maires évoquent de manière récurrente la désertification et le départ des jeunes, qui, à la fin de leurs études, ne reviennent pas au pays. Dans cette perspective, on comprend que les stations apparaissent comme une opportunité de valoriser les terrains à construire et de maintenir la population sur place. L'étendue du domaine skiable semble un critère décisif pour les professionnels du tourisme cherchant à séduire les voyagistes, mais les élus disposent d'autres arguments intéressants aussi les exploitants de domaines. Leur rôle est en effet capital lors du montage et du dépôt $\mathrm{du}$ «dossier d'unité touristique nouvelle», indispensable à l'installation des promoteurs immobiliers et des remontées mécaniques. Ils disposent en outre des ressources réglementaires nécessaires au développement de l'immobilier touristique et à la construction des lits nécessaires à la rentabilité des stations sur leur commune. Les élus signataires des permis de construire délimitent des zones constructibles et exploitables par les promoteurs immobiliers, et travaillent à l'adhésion de la population.

« Et donc le lancement d'une station, c'est un peu l'ouverture vers une économie de marché pour arriver à faire comme on dit toujours "du travail au pays". Alors ça veut 
dire quoi devenir une station ? Ça veut dire être capable d'offrir un certain nombre de prestations. D'abord du logement: "Venez chez nous, on peut vous accueillir." "Oui, mais qu'est-ce que je peux faire?" "Vous allez pouvoir faire du ski. Ski de piste et ski de fond." [...] Ce qui veut dire qu'on passe d'un télésiège deux places à une télécabine. Donc du coup, on peut accroître notre capacité d'hébergement et donc on peut mettre en place des programmes immobiliers » (un élu, 2008).

De leur côté, les stations de sports d'hiver représentent pour les communes une manne d'emplois dont une partie est directement liée à l'exploitation des pistes et du domaine skiable. C'est le cas des perchmans, des dameurs, des moniteurs de ski ou des guides de montagne, qu'il s'agisse de leur activité principale ou d'une activité secondaire. L'existence d'une station permet également la création d'emplois moins directement liés à l'exploitation des remontées et du domaine. L'hôtellerie, la restauration ainsi qu'un grand nombre de commerces se développent dans et autour de la station. Les stations participent enfin au financement de certains équipements communaux et de certaines activités. Le développement économique par le tourisme d'hiver, s'il contribue à maintenir la population au pays et à attirer de nouveaux résidents, participe largement à l'enrichissement de ces communes de Tarentaise qui disposent parfois de budgets sans commune mesure avec celui du parc. Dans ce contexte, on comprend que les élus qui bénéficient de terrains intéressants puissent céder aux avances des stations cherchant à étendre leur domaine d'activité pour mieux valoriser les kilomètres de pistes qu'elles sont susceptibles de mettre à la disposition des touristes.

Alors que cet arrangement entre élus et stations contribue à l'augmentation des prix du foncier bâti, les agriculteurs les plus éloignés des domaines skiables voient se rapprocher progressivement les aménagements associés à l'extension de ces domaines et pointent du doigt l'incompatibilité entre le rythme de leurs activités et celui de l'activité touristique. Les agriculteurs qui pratiquent ici une agriculture d'élevage produisant du lait et du fromage - commercialisé notamment sous l'AOC Beaufort - sont ainsi progressivement repoussés vers des zones plus éloignées, plus pentues et accidentées, plus risquées et avalancheuses, et perdent ainsi des journées de pâturage. L'arrangement entre exploitants et élus se traduit ainsi par des conditions de travail plus difficiles pour les agriculteurs, qui doivent se rendre sur des terrains situés en altitude, se trouvant ainsi confrontés au manque de végétation pour leurs bêtes.

« La pression foncière est importante. Parce que là, à 1550 où on démarre l'alpage, on a notre premier chalet de fabrication et une cave d'affinage qu'on a construite il y a quatre ans pensant être tranquilles et au bout du monde, puisqu'on était vraiment retirés de la station. Et depuis quatre ans, on nous a planté juste à côté un HLM. Donc ça, c'est déjà une journée de pâturage en moins. Au-dessus, il vient de se vendre 4 hectares de pâturage. Et puis petit à petit, on va être obligés de partir. À 3 h du matin on démarre les engins, c'est vrai que ça fait du bruit, les vaches, les sonnettes, etc. Alors on va de plus en plus haut. Enfin, comme on construit jusqu'à 2000 maintenant, on se demande jusqu'où il va falloir aller parce que la végétation, elle a aussi une limite » (groupement pastoral, 2008).

On comprend alors que les agriculteurs tiennent fréquemment des propos relativement critiques à l'égard de leurs élus dont les arbitrages leur apparaissent être systématiquement en faveur des stations. Les agriculteurs ne pointent pas simplement la passivité des élus qui ne soutiennent pas assez les pratiques pastorales, ils dénoncent aussi la pratique des maires qui mobilisent leur expertise réglementaire et leur légitimité d'élus pour récupérer et mettre à disposition des stations les terrains les plus intéressants.

Ainsi, la régulation du système d'action en Tarentaise apparaît d'abord structurée par l'arrangement entre les responsables des domaines skiables et les élus des différentes communes de la vallée. Nous devons désormais analyser l'activité des agents du parc pour administrer leur secteur.

\section{De la police de l'environnement à l'hétérogénéité des arrangements ponctuels}

Les agents de terrain évoquent aisément les effets nuisibles des stations sur la biodiversité. Les travaux de terrassement nécessaires à l'aménagement des pistes et à l'installation des gares d'arrivée de télésiège détruisent non seulement les espèces floristiques qui se trouvent sur le tracé mais dérangent aussi la faune. Ce ne sont pas les ongulés les plus menacés mais plutôt les galliformes, leur mortalité due aux collisions avec les câbles des remontées étant relativement importante. Ces aménagements ont par ailleurs des conséquences plus profondes et plus durables sur les paysages et les milieux de vie de ces espèces. C'est cette idée que mettent en avant les agents lors des entretiens en nous montrant, à l'aide d'une carte, le caractère expansionniste des remontées mécaniques quadrillant de manière toujours plus serrée le territoire, mais aussi le caractère invasif d'un tourisme de masse encourageant la course à l'enneigement artificiel et posant la question de la gestion des déchets. Néanmoins, les agents reconnaissent dans le même temps ne disposer que de peu de ressources pour endiguer l'expansionnisme des domaines. Si le budget du parc apparaît bien limité au regard des flux financiers dégagés par l'industrie touristique, les agents de terrain concentrent en priorité leur travail autour des deux missions traditionnelles du parc. Il s'agit, d'abord, de sanctionner les infractions au code de l'environnement et au décret de 1963. À travers ces missions de police, il n'est 


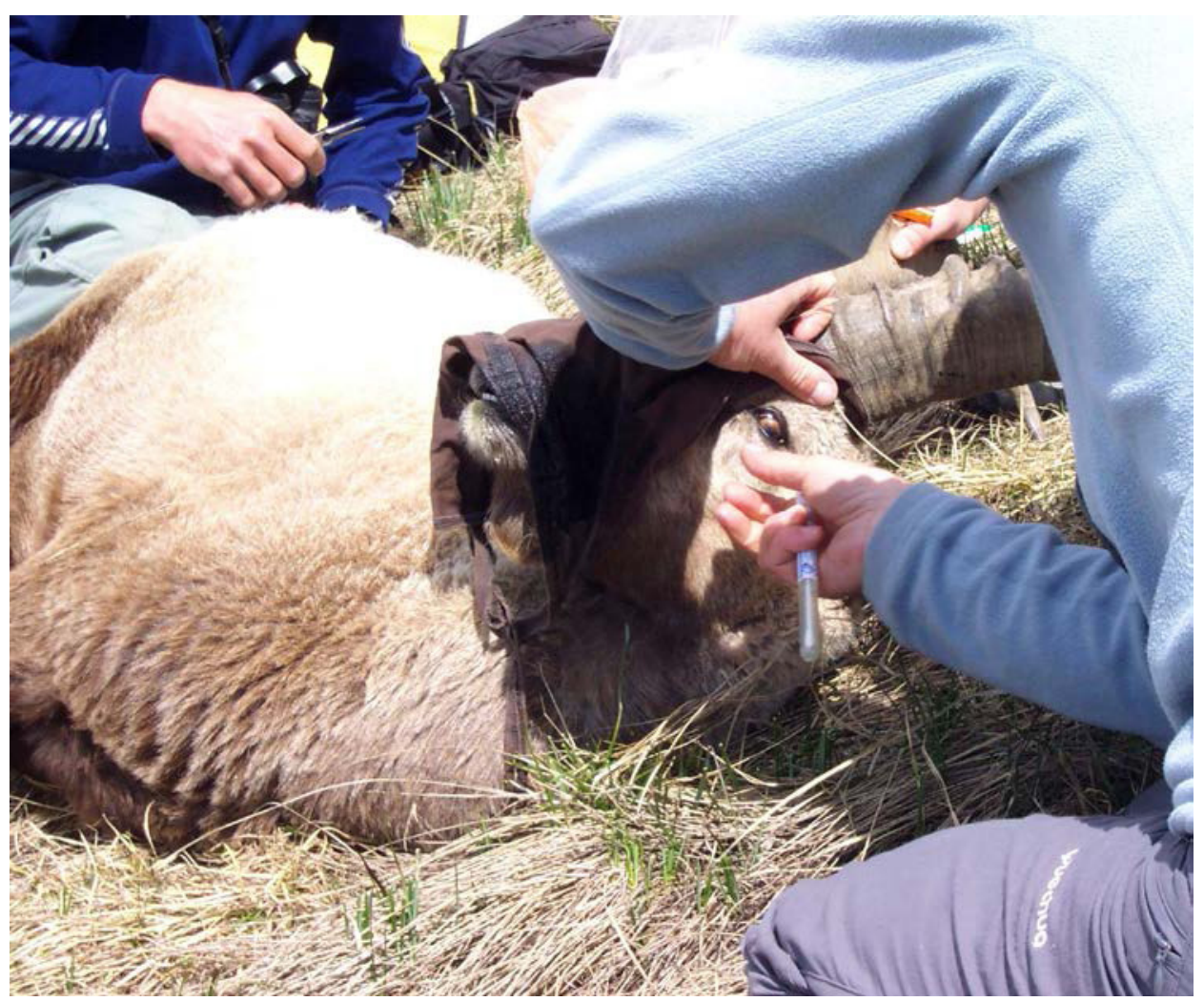

Photo 1. Comptage bouquetins, 2008 (Photo : Thomas Debril).

pas tant question de surveiller les éventuels débordements des stations que de réaliser une police de la chasse, de la pêche ou de la circulation renvoyant aux incidents en matière de pollution. Il s'agit, ensuite, d'assurer la mission de veille écologique consistant à se déplacer sur le terrain pour assurer un suivi floristique et faunistique $\mathrm{du}$ milieu. Il semble bien ici que le peu de prise des agents sur les stations se traduise par un surinvestissement dans les activités naturalistes, correspondant le plus souvent aux motivations premières des agents. Leur travail consiste alors à suivre la reproduction des rapaces, réaliser des comptages de bouquetins ou des prospections flore sur les espèces jugées prioritaires par leur direction (Photo 1).

Si les agents du parc et les personnels des stations n'entretiennent finalement que très peu de relations, les agents développent toutefois des actions, ponctuelles et hétérogènes, avec les autres acteurs du territoire au premier rang desquels figurent les agriculteurs. Si quelques agriculteurs profitent de la présence des touristes drainés par les stations pour écouler directement une partie de leurs productions, la majeure partie d'entre eux paient ici les coûts de la coopération entre stations et élus, et développent un discours positif à l'égard des personnels du parc dont ils acceptent de prendre en compte les enjeux environnementaux. Il s'agit par exemple de poser une clôture pour éviter le surpâturage d'une zone déjà surexploitée et permettre l'éventuel retour de la flore. Il est vrai que la pose des clôtures et que le confinement des animaux évitent non seulement le vagabondage des brebis dans les moraines et les incidents associés au dérochement, mais aussi le travail parfois important qu'il faut déployer pour rassembler les animaux. De leur côté, les agents du parc acceptent d'apporter quelques services comme l'héliportage ou le financement de clôtures. En facilitant le travail des agriculteurs relégués sur des zones difficiles, le parc maintient une activité pastorale sur des zones qui ont tendance à être abandonnées. Les agriculteurs qui participent au maintien de la zone ouverte et à son entretien par le pâturage profitent des dédommagements de l'administration. Les agriculteurs voient ainsi dans les enjeux environnementaux portés par le parc moins une menace de contraintes supplémentaires qu'une opportunité de continuer à faire vivre ces alpages de faible valeur qui ont tendance à être délaissés.

Les agents entretiennent par ailleurs des relations avec des accompagnateurs, professionnels indépendants qui, sans être guides, organisent des randonnées en montagne. Le parc apporte à certains accompagnateurs un label et une visibilité facilitant leur insertion dans l'économie touristique. Que ce soit par l'intermédiaire des offices de tourisme, de la revue éditée par le parc ou plus simplement et plus directement à l'occasion des 
randonnées que les agents proposent à la clientèle, les personnels du parc font connaître l'activité des accompagnateurs et proposent leurs prestations. De leur côté, les accompagnateurs sensibilisent les touristes au caractère exceptionnel d'un territoire nécessitant une protection particulière, reprennent le discours et expliquent les actions développées par le parc. Les accompagnateurs évoquent non seulement l'expertise scientifique développée par les chargés de mission, mais aussi son action politique ou gestionnaire en matière de protection de la nature auprès $\mathrm{d}^{\prime}$ une population parfois très jeune et avec laquelle certains agents reconnaissent avoir du mal à communiquer. Les accompagnateurs contribuent ainsi à la bonne image du parc auprès des populations et à l'amélioration des relations entre le parc et les élus relativement «friands » de ce type d'animation dans les écoles.

De même, les exploitants des petites «stations village » n'adoptent pas du tout le même discours que leurs homologues des grandes " usines à ski » à l'égard du parc. Loin de considérer les préoccupations environnementales du parc comme des contraintes supplémentaires, ils y voient au contraire l'occasion de se différencier de la concurrence et de proposer une autre qualité de séjour, renvoyant au caractère familial d'une station respectueuse de l'environnement. Le parc contribue à l'image de marque de ces petits domaines, soutient leur stratégie de différenciation et facilite leur positionnement sur le marché très concurrentiel du tourisme. Société d'exploitation et agents du parc se retrouvent ainsi autour d'un tourisme qui réaffirme les enjeux associés au respect de la nature.

En définitive, l'arrangement que nous avons pu observer entre exploitants de domaines skiables et maires de communes, s'il date des années 1960, apparaît encore largement structurant dans l'organisation socioéconomique de la Tarentaise. C'est bien cet arrangement entre le marchand et le politique qui semble à l'origine de cette relative étanchéité de la Tarentaise à l'égard du parc. Alors que les gérants des domaines skiables et les agents du parc semblent avoir des enjeux largement opposés, ces deux types d'acteurs ne semblent pas entretenir des relations importantes. C'est bien la stratégie du chacun chez soi qui semble primer, le parc ne disposant finalement que de peu de ressources pour modifier les pratiques de ces interlocuteurs.

Pour autant, il ne faudrait pas en conclure que les agents du parc sont peu actifs. Bien au contraire, ils compensent leur relative impuissance et les conséquences environnementales associées au développement de l'industrie touristique par un engagement accru dans les activités scientifiques, et par des arrangements ponctuels avec les acteurs qui vivent autour des stations et qui paient, pour une part, les coûts de cette régulation. C'est bien ce que nous montre la diversité des partenariats qu'ils parviennent à nouer avec les agriculteurs (Druguet, 2007) dans ce qui peut s'apparenter ici à une forme de réhabilitation des activités pastorales (Laslaz, 2006), avec les petites stations de ski ou les accompagnateurs de montagne. Il ne s'agit donc pas d'ironiser sur ces actions qui, certes, n'enrôlent pas les acteurs les plus puissants de ce système, mais de reconnaître qu'elles sont partie intégrante des missions des parcs travaillant aussi bien à la protection des espaces et à la conservation des milieux qu'au développement des activités et à l'animation des territoires.

\section{La Maurienne, porosité du territoire et action au cœur}

L'activité du parc au sein de la Maurienne apparaît bien différente. Il est vrai que cette vallée est loin de disposer des mêmes infrastructures puisqu'elle ne compte que 71 remontées mécaniques sur les 520 de la Vanoise et ne présente pas les mêmes capacités d'hébergement car seulement près de $18 \%$ de la capacité d'accueil touristique marchand se trouve en Maurienne. La relative défiance à l'égard du parc semble plutôt renvoyer ici aux arrangements que l'on peut observer entre les maires des communes et les agriculteurs travaillant au sein d'exploitations familiales et valorisant par l'intermédiaire de la coopérative laitière de la vallée le fromage Beaufort et le bleu de Termignon, issus des vaches tarine et abondance. Alors qu'en Tarentaise les troupeaux sont regroupés sur de grands alpages communaux loués à des groupements pastoraux avec des effectifs gardés, les agriculteurs de la Maurienne travaillent le plus souvent sur des alpages privés et des prairies de fauche en altitude non gardées. Les agriculteurs évoquent ici, assez aisément, le caractère intrusif du parc et apparaissent particulièrement rétifs à ses injonctions environnementales. On peut du même coup s'interroger au sujet des marges de manœuvre dont disposent les agents pour administrer leur territoire et, notamment, influer sur les pratiques agricoles.

\section{Défiance à l'égard du parc et cohabitation avec l'économie touristique : la posture agricole}

Les agriculteurs de haute Maurienne n'adoptent pas le même point de vue que leurs homologues de Tarentaise à l'égard du parc. Alors que les premiers souffrent du développement touristique soutenu par les exploitants des domaines skiables et les maires, et apparaissent favorables aux mesures de protection environnementale, les seconds sont au contraire relativement opposés aux agents et tout particulièrement à la direction du parc. Les restrictions imposées par celui-ci en matière d'utilisation des pistes pour se rendre aux alpages semblent cristalliser une grande part de leurs 


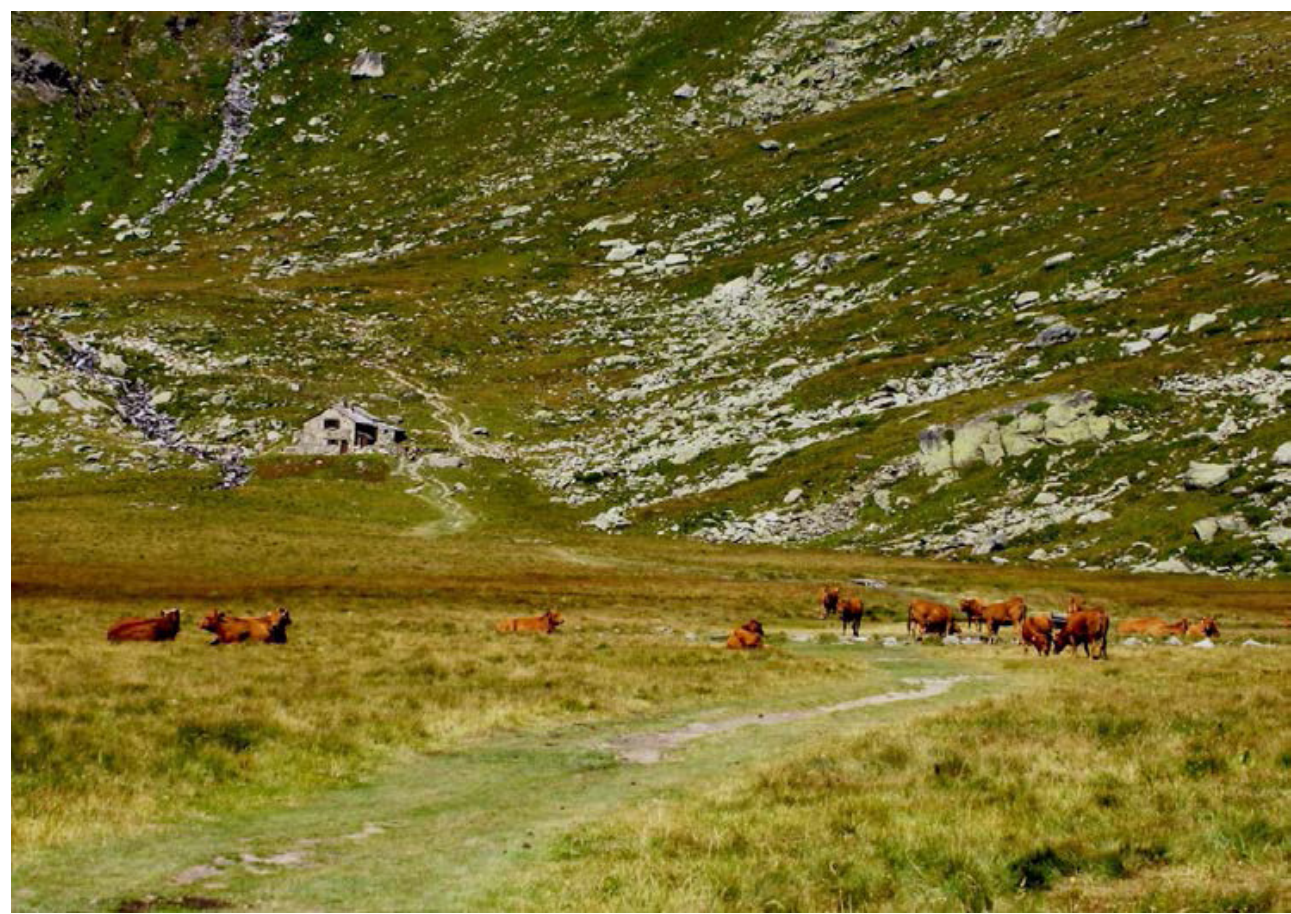

Photo 2. Pratiques pastorales en alpage, 2010 (Photo : Gérard Debril).

reproches. La création du parc apparaît ainsi associée à l'apparition de nouvelles contraintes qui remettent en cause le caractère traditionnel des pratiques agricoles et nourrissent un fort sentiment de dépossession (Photo 2). L'irritation des agriculteurs est attisée par les recommandations des agents qui se mêleraient de gestion pastorale alors même qu'ils méconnaissent les modes d'exploitation agricole qui ont cours sur le territoire depuis de nombreuses années, la manière dont fonctionnent les entreprises agricoles et les conditions relativement difficiles qui se rapportent à cette activité en montagne.

« Je suis propriétaire de mon terrain et depuis que le parc est venu, chaque année, ou chaque dix années, il y a une couche qui s'empile soit pour aller dans mon alpage, soit pour autre chose. Au début tout était libre, ensuite on a commencé à dire, $t^{\prime}$ as pas le droit de ceci pour utiliser la route, pour aller à Entre-deux-Eaux, c'est des laissez-passer, ensuite il faut aller les chercher à la mairie, ensuite il fallait donner ton nom, après c'est combien de générations, après c'est si tu fabriques sur place. Moi je suis propriétaire, je suis chez moi. Alors quand on ne connaît rien à un territoire, qu'on ne connaît rien à une exploitation agricole et qu'on vient parce qu'on a appris que les oiseaux ils chantaient le matin à je ne sais pas quelle heure, et qu'on vient vous apprendre ce qu'il faut faire chez vous, il y a un moment voilà » (un agriculteur, 2008).

Le parc est non seulement perçu comme un problème limitant leur activité mais aussi comme un acteur ne proposant pas de réelles solutions aux difficultés qu'il soulève. Il est vrai que l'opposition entre les pratiques agricoles et les enjeux environnementaux peut apparaître relativement indépassable sur un certain nombre de dossiers. C'est le cas par exemple pour les invasions de rumex auxquelles sont confrontés les agriculteurs qui, pour s'en débarrasser, utilisent des pesticides difficilement acceptables pour les chargés de mission. Les solutions mécaniques respectueuses de l'environnement apparaissent insuffisantes pour combattre ces plantes nitrophiles qui profitent des sols fortement chargés en azote. Si les chargés de mission reconnaissent alors l'importance d'une gestion en amont et de long terme incitant les agriculteurs à ne pas laisser les machines à traire trop longtemps au même endroit, ils reconnaissent dans le même temps les difficultés qu'ils éprouvent à faire passer ce message. C'est bien cette absence de propositions concrètes que pointent encore du doigt les agriculteurs à propos de la controverse au sujet des contaminations entre faune sauvage et faune domestique, autour des produits nécessaires au traitement des animaux. Le parc souhaiterait interdire l'utilisation de certains produits sans toutefois proposer des solutions de remplacement ou sans se soucier de la faisabilité de leur mise en œuvre. Si le parc propose le parcage des animaux comme solution pour trancher la controverse autour des contaminations, il n'envisage pas les conséquences de ce dispositif pour les agriculteurs, le manque à gagner que constitue le gardiennage et les coûts qui y sont inévitablement associés. Plus que cela, la clôture provoque d'autres problèmes au premier rang desquels figure le piétinement contre lequel le parc entend lutter. C'est 
bien l'incohérence des recommandations du parc et la stérilité des interminables réunions que les agriculteurs regrettent ici.

Si les agriculteurs de la Maurienne ne considèrent pas le parc comme un allié potentiel dans la défense de leurs intérêts, ils ne voient pas non plus les stations comme une menace dans leurs pratiques pastorales. Les stations de ski de la Maurienne, loin d'offrir le même kilométrage de pistes que leurs homologues de Tarentaise, ne représentent pas une réelle menace en matière de réduction des prés de fauche et plus généralement du foncier. Alors que les pistes de ski fournissent un accès privilégié aux agriculteurs qui doivent faire monter leurs troupeaux dans les alpages l'été, les vaches participent à l'entretien du paysage en broutant l'herbe et contribuent ainsi à un meilleur enneigement. Activités touristiques et activités pastorales semblent présenter ici une relative complémentarité. Plutôt que de dénoncer l'expansionnisme des stations et relever la recrudescence des conflits d'usages associés à l'affluence des touristes, les agriculteurs préfèrent mettre en avant les avantages associés à la présence de ces stations qui fournissent de l'emploi et drainent une clientèle leur permettant de développer une vente directe rémunératrice. Dans cette perspective, agriculteurs et stations prennent leur distance à l'égard du parc et plus précisément à l'égard de sa direction. $C^{\prime}$ est bien ce que l'on peut observer dans le soutien que les élus apportent aux agriculteurs contre la direction du parc. Nous retrouvons alors les critiques prenant comme point d'appui l'importance des dossiers de compensation et la lourdeur d'un établissement public privilégiant trop fréquemment les enjeux environnementaux au détriment des enjeux économiques.

\section{Entre coercition et pédagogie : la négociation des règles du jeu}

Les agents du parc de haute Maurienne ne parlent pas de leur travail dans les mêmes termes que leurs homologues de Tarentaise. En effet, il ne s'agit pas tant de contourner les stations en travaillant en périphérie du système que de nouer un contact étroit avec la population agricole. L'activité des agents nous montre les efforts qu'ils déploient pour éviter la posture du chacun chez soi, défaire les discours caricaturaux qui mettent en scène la figure du bureaucrate d'un côté et celle du pollueur de l'autre. Les agents négocient ici la prise en compte des enjeux environnementaux en relâchant l'application systématique de la règle. C'est bien ce qu'évoquent certains agents reconnaissant qu'il ne s'agit pas de fermer les yeux sur les infractions mais de faire preuve de souplesse à l'égard de la réglementation à laquelle sont régulièrement confrontés les agriculteurs. Une grande part de l'expertise de ces agents assermentés consiste à négocier l'application du règlement et à choisir le moment à partir duquel on prolonge la discussion par la verbalisation d'une pratique spécifique. Nous retrouvons sur ce point l'inévitable autonomie des agents administratifs de terrain (Lipsky, 1980) et le pouvoir discrétionnaire dont ils disposent à l'égard de leurs ressortissants (Worms, 1966). Cette capacité d'arbitrage entre les différents événements auxquels ils sont confrontés constitue de fait une grande part de l'intérêt de leur métier.

«Fermer les yeux, c'est pas le cas, par contre, on a des alternatives. Voilà. C'est facile de verbaliser une pauvre madame en vacances ici qui va cueillir deux fleurs, 900 balles. Ce qui est moins facile, c'est quand on est un paysan et qu'on a des locaux ici en zone quasi rouge. $C^{\prime}$ est là que le réglementaire pour moi personnellement, c'est un outil. Des fois ça sert, des fois ça nous dessert. C'est toute la difficulté $d$ 'un agent de terrain qui est assermenté pour faire respecter une loi, mais à des moments vaut mieux aller voir la personne et dire "voilà, on a tel souci, nous ce qu'on souhaite c'est que ça n'aille pas plus loin, on aimerait que... Qu'est-ce que vous en pensez ? On s'arrange". Et des fois y'a moyen, des fois pas, faut verbaliser. Ce qui est difficile c'est décider quand est-ce qu'on verbalise, ou qu'on dit "vous vous arrêtez, on corrige, on recommence" » (un agent, 2008).

Les agents qui prennent leurs distances à l'égard de la règle formelle et ne considèrent plus nécessairement celle-ci comme un point de référence obligé doivent alors composer avec la diversité des positionnements plus ou moins répressifs ou permissifs qui coexistent au sein de l'établissement, composer avec les différentes manières de concevoir leur travail. Établir une position commune assurant la cohérence des démarches demande un véritable investissement de la part de ces agents. La tâche apparaît d'autant plus difficile que les recrutements ont été ouverts à un concours national, ce qui a entraîné l'arrivée d'un personnel hétérogène. Les agents les plus anciens évoquent à ce sujet le personnel issu des corps de police comme l'ONC qui, en adoptant une posture trop stricte à l'égard de l'application de la règle, vient remettre en cause la construction de ces nouveaux liens qu'ils cherchent à établir avec les agriculteurs. Dans ce contexte, les réunions des différents agents du parc apparaissent comme un moment décisif où sont harmonisés les points de vue. Il s'agit alors de créer à cette occasion une représentation partagée des problèmes environnementaux comme des solutions à préconiser, $\mathrm{d}$ 'adopter une démarche commune et, finalement, de se doter de nouvelles règles. C'est bien ici que se travaille l'articulation entre la personnalisation de réponses sur mesure produites par le parc et la cohérence en interne de l'ensemble des actions déployées par les agents sur l'ensemble du secteur. C'est ici que se négocie le compromis entre l'application stricto sensu de la règle permettant de traiter de manière standardisée de nombreux cas et 
un traitement plus personnalisé et coûteux des dossiers dans ce qui peut s'apparenter à une "production juridique en continu » (Lascoumes, 1990) et par laquelle sont retravaillés les cadres de l'action environnementale (Mormont, 2006).

\section{Conclusion}

L'activité des agents du parc de la Vanoise nous oblige à renoncer à l'opposition simpliste entre la représentation juridique d'une politique publique sans hommes consacrant le pouvoir réglementaire de l'administrateur d'un côté, et la représentation sociologique des hommes sans politique publique réifiant les stratégies de détournements des administrés de l'autre. Les agents du parc nous présentent plutôt deux formes négociées d'administration du territoire, attentives à la diversité des enjeux portés par l'hétérogénéité des acteurs présents sur le terrain. Nous avons voulu montrer que si l'action menée par la direction du parc prend finalement deux formes différentes en Maurienne et en Tarentaise, c'est que les agents du parc chargés de sa mise en œuvre sur le terrain sont confrontés à des formes d'organisation socioéconomique qui n'offrent pas les mêmes prises à leur mission environnementale et les obligent à négocier différemment leur travail sur le territoire. À la relative étanchéité de la Tarentaise renvoyant à l'arrangement entre maire et station correspond un surinvestissement dans l'inventaire scientifique et l'action pédagogique. À la relative porosité de la Maurienne renvoyant au plus grand équilibre entre activités touristiques et agricoles correspond une action des agents plus engagée dans la négociation des enjeux environnementaux. Dans ce contexte, le comportement différencié des agents de terrain fait bien davantage référence à l'organisation spécifique du territoire qu'ils administrent qu'à l'intériorisation de normes et de valeurs particulières. Tout se passe finalement comme si nous avions, d'un côté, des agents transparents à l'égard de leur direction, mais agissant pour l'essentiel à la marge de leur système d'action et, de l'autre, des agents plus impliqués dans l'animation du territoire mais plus opaques aussi dans leurs modes de fonctionnement.

Pour autant, le calibrage des interventions des agents de terrain selon l'organisation du territoire auquel ils ont à faire interroge directement la cohérence des pratiques comme la légitimité des décisions (Lascoumes et Le Bourhis, 1998) prises par ces agents. La prégnance des enjeux environnementaux dans l'activité de ces personnels animant de manière contrastée leur territoire provoque en effet d'importantes polémiques, au sein de la direction, sur la nature des problèmes à traiter en priorité. Alors que certains chargés de mission reconnaissent qu'il leur manque une expertise scientifique pour mesurer l'impact des pratiques, notamment pastorales, sur les milieux et que c'est bien dans ce sens que des efforts humains et financiers doivent être consentis, d'autres, à l'inverse, mettent en avant la disproportion des moyens scientifiques mobilisés pour réaliser les études d'impact et le coût de ces diagnostics d'alpages. Les divergences entre chargés de mission portent ensuite sur les solutions et la nature des propositions à adopter. Alors que l'autorisation accordée à l'élargissement d'une piste existante peut être vue par certains comme une preuve de laxisme de la part du parc, d'autres voient dans cet aménagement une solution intelligente et préfèrent souligner les compensations accordées par l'alpagiste.

L'institutionnalisation des formes d'action collective que nous donne à voir l'action publique territorialisée du parc (Duran et Thoenig, 1996) se traduit ainsi par d'importantes controverses en son sein qui ne sont pas sans conséquence sur les agents de terrain. Ces derniers se plaignent d'abord de la multiplication des réunions auxquelles ils sont invités et lors desquelles ils peuvent constater la diversité des points de vue qui coexistent au sein de leur direction. Si l'étendue des missions du parc légitime la diversité des actions des agents sur le terrain, elle suppose aussi un important travail de coordination en interne. Ce sont bien les coûts associés à cette coordination que les agents pointent du doigt lorsqu'ils évoquent la bureaucratisation de leur établissement qui remet en cause les sorties sur le terrain pour lesquelles ils sont fréquemment entrés dans la profession, nous rappelant cette professionnalisation en tension des métiers de la nature (Granjou et al., 2010). La multiplicité des délibérations au sein de la direction conduit non seulement à un manque de réactivité à l'égard des problèmes qu'ils font remonter mais aussi à un manque de cohérence quant aux arbitrages qui sont finalement arrêtés. Les coûts du sur-mesure sont alors supportés par les agents de terrain, au contact permanent des acteurs locaux qui suivent fréquemment de près les altercations avec le parc. Pour autant, ces agents loin de jouer la stratégie du repli bureaucratique (Mouhanna, 2001) sélectionnent dans les situations problématiques celles qu'ils sont en mesure de résoudre pour renvoyer les difficultés qu'ils rencontrent vers le siège et ses chargés de mission. Les coûts de la négociation locale des agents apparaissent ainsi transférés sur la direction qui rencontre quelques difficultés dans la logique partenariale qu'elle souhaite mettre en place. Cette logique ne fabrique pas simplement du sens commun et des solutions acceptables au sein des territoires, elle permet aussi aux agents de rester 
en contact avec le terrain et de maintenir leur crédibilité vis-à-vis des acteurs locaux.

\section{Références}

Boncœur, J., Noël, J.-F., Sabourin, A., Tsang King Sang, J., 2007. La gouvernance des aires marines protégées : le projet de parc marin en Iroise, un exemple de processus participatif ?, Monde en Développement, 2, 138, 77-92.

Cibien, C., 2006. Les réserves de biosphère: des lieux de collaboration entre chercheurs et gestionnaires en faveur de la biodiversité, Natures Sciences Société, 14, 84-90.

Crosnier, C., 2006. Biodiversité et pertinence des pratiques locales dans la réserve de la biosphère des Cévennes, Revue Internationale des Sciences Sociales, 1, 187, 159-168.

Crozier, M., Friedberg, E., 1977. L'Acteur et le Système, Paris, Le Seuil.

Delsaut, M., Meur-Férec, C., 2009. La réserve naturelle nationale du platier d'Oye : analyse de vingt ans de conflits (1987-2007), Natures Sciences Sociétés, 17, 2, 155-160.

Duran, P., 1993. 1. L'environnement une affaire d'État? Le parc national des Pyrénées occidentales ou les difficultés de la négociation institutionnalisée, Annuaire des Collectivités Locales, 13, 5-32

Duran, P., Thoenig , J.C., 1996. L'État et la gestion publique territoriale, Revue Française de Science Politique, 46, 4, 580-623.

Druguet, A., 2007. Concilier agriculture et conservation d'un paysage de terrasses à la périphérie du parc national des Cévennes, Géocarrefour, 82, 4, 199-207.

Fortier, A., 2008. Quand la concertation produit de l'évitement : la mise en œuvre de Natura 2000 en région NordPas-de-Calais, Développement Durable et Territoires, 3, 2 (online: http://developpementdurable.revues.org/4602).

Giran, J.-P., 2003. Les parcs nationaux: une référence pour la France, une chance pour ses territoires. Rapport au Premier ministre, Paris, La Documentation française.

Granjou, C., Mauz, I., Cosson, A., 2010. Les travailleurs de la nature: une professionnalisation en tension, SociologieS [en ligne] (online : http://sociologies.revues.org/index3296. html).
Jobert, A., 1998. L'aménagement en politique: ou ce que le syndrome NIMBY nous dit de l'intérêt général, Politix, 11, 42, 67-92.

Lascoumes, P., 1990. Normes juridiques et mise en œuvre des politiques publiques, L'Année Sociologique, 40, 43-71.

Lascoumes, P., Le Bourhis, J.-P., 1998. Le bien commun comme construit territorial : identités d'action et procédures, Politix, 11, 42, 37-66.

Laslaz, L., 2006. Terre d'élevage ou « nature préservée » en zone centrale des parcs nationaux français des Alpes du Sud ?, Méditerranée [en ligne] (online: http:/ / mediterranee. revues.org/index462.html).

Lipsky, M. 1980. Street-Level Bureaucracy: Dilemmas of the Individual in Public Services, New York, Russel Sage.

Mathevet, R., Thompson, J., Delanoë, O., Cheylan, M., Gil-Fourrier, C., Bonnin, M., 2010. La solidarité écologique : un nouveau concept pour une gestion intégrée des parcs nationaux et des territoires, Natures Sciences Sociétés, $18,4,424-433$.

Mauz, I., 2003. Histoire et mémoires du parc national de la Vanoise, 1921-1971: la construction, Grenoble, Revue de Géographie Alpine.

Milian, J., 2007. Le dilemme entre développement et protection dans les montagnes du Maroc: le cas des parcs du Moyen Atlas, Géocarrefour, 82, 4, 177-186.

Mormont, M., 2006. Conflit et territorialisation, Géographie, Économie, Société, 8, 299-318.

Mouhanna, C., 2001. Faire le gendarme: de la souplesse informelle à la rigueur bureaucratique, Revue Française de Sociologie, 42, 1, 31-55.

Muller, P., 2005. Esquisse d'une théorie du changement dans l'action publique : structures, acteurs et cadres cognitifs, Revue Française de Sciences Politiques, 55, 1, 155-187.

Musselin, C., 2005. Sociologie de l'action organisée et analyse des politiques publiques : deux approches pour un même objet ?, Revue Française de Science Politique, 55, 1, 51-71.

Nay, O., Smith, A., 2002. Le Gouvernement du compromis : courtiers et généralistes dans l'action politique, Paris, Economica.

Selmi, A., 2006. Administrer la nature: le parc national de la Vanoise, Paris, Éditions de la Maison des Sciences de l'Homme, Éditions Quae.

Worms, J.-P., 1966. Le préfet et ses notables, Sociologie $d u$ Travail, 8, 3, 249-275.

Accepté le 9 septembre 2013. 